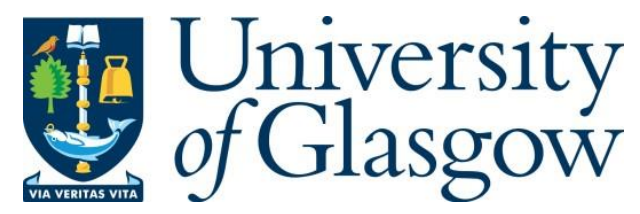

Jull, A.J.T., Scott, E.M. and Bierman, P. (2015) The CRONUS-Earth inter-comparison for cosmogenic isotope analysis. Quaternary Geochronology, 26, pp. 3-10.

There may be differences between this version and the published version. You are advised to consult the publisher's version if you wish to cite from it.

$\underline{\text { http://eprints.gla.ac.uk/229024/ }}$

Deposited on: 20 January 2021

Enlighten - Research publications by members of the University of Glasgow http://eprints.gla.ac.uk 


\title{
The CRONUS-Earth inter-comparison for cosmogenic isotope analysis
}

\author{
A. J. T. Jull ${ }^{1,2,+}$, E. M. Scott ${ }^{3}$ and P. Bierman ${ }^{4}$
}

1. NSF Arizona AMS Laboratory, University of Arizona, Tucson, Arizona 85721 USA.

2. Department of Geosciences, University of Arizona, Tucson, Arizona 85721 USA

3. School of Mathematics and Statistics, University of Glasgow, Glasgow, Scotland

4. Department of Geology and Rubenstein School of the Environment and Natural Resources, University of Vermont, Burlington, Vermont 05405 USA

+Corresponding author. Email: jull@email.arizona.edu; tel. +15206216816

\section{Abstract}

As part of the NSF-funded program CRONUS-Earth, a series of natural reference materials for in situ produced ${ }^{26} \mathrm{Al},{ }^{10} \mathrm{Be},{ }^{14} \mathrm{C}$, and ${ }^{36} \mathrm{Cl}$ were prepared and circulated to United States, Australian, and European laboratories for analysis to explore the comparability of results from the different laboratories and generate preliminary consensus values for a range of reference material. Such reference materials, which did not exist for these isotopes, assist laboratories in independently assessing quality and are useful to quantify precision and accuracy. Currently, most researchers report only internal analytical uncertainties for all results. While researchers have acknowledged the need for realistic inter-laboratory uncertainties for in situ produced cosmogenic isotopes, few previous studies have addressed this issue. Two samples (denoted $\mathrm{A}$ and $\mathrm{N}$ ) were provided for ${ }^{26} \mathrm{Al},{ }^{10} \mathrm{Be}$ and in situ ${ }^{14} \mathrm{C}$ analysis, one from the Antarctic, high in ${ }^{26} \mathrm{Al}$ and ${ }^{10} \mathrm{Be}$ and the other from Australia, lower in both ${ }^{26} \mathrm{Al}$ and ${ }^{10} \mathrm{Be}$. Both samples were prepared to quartz at the University of Vermont. For each sample, results have been summarised in terms of the mean reported concentration, standard deviation both between (inter) and within (intra) laboratories to describe inter- and intra-laboratory variability. Coefficients of variation (CoV) expressed as a percentage of the mean are also reported. For in-situ ${ }^{14} \mathrm{C}$, a small number of laboratories reported results, so they are summarised separately. Initial uncorrected results for samples $A$ and $N$ showed significant variation (greater than $8 \% \mathrm{CoV}$ ) in results, due to differences in standardization. When corrected to a common basis, the $\mathrm{CoV}$ was $2.9 \%$ for ${ }^{10} \mathrm{Be}$ measurements of sample A (high concentration) and to $4.1 \%$ for sample $\mathrm{N}$ (lower concentration), which is closer to typical cosmogenic samples. ${ }^{26} \mathrm{Al}$ measurements had greater variation; a CoV of $4.9 \%$ was achieved for sample A (high concentration) but for the lower concentration sample $\mathrm{N}$, the CoV was $10.1 \%$.

Highlights:

- We report on an intercomparison study between different CRONUS laboratories

- We find high-concentration ${ }^{10} \mathrm{Be}$ samples give interlab results within $<5 \%$ error

- In the best cases $<3 \%$ error is achieved

- We find higher errors for ${ }^{26} \mathrm{Al}$ at $\sim 5 \%$ and $10 \%$ for lower-level samples

- A series of references materials have been characterised

Keywords:cosmogenic nuclides; intercomparison; errors; reference material; consensus values 


\section{Introduction}

Understanding and quantifying measurement uncertainty is an important and essential step in applied isotope analysis. Every time that an analytical isotope analysis is repeated, even under identical conditions, a different result will be obtained. Laboratories usually make only one measurement on a sample, but if repeated measurements are made, this scatter can be quantified and thus laboratories can provide an estimate of the analytical uncertainty that reflects the range of values (or the spread) in results that would likely have been obtained were the measurement to be repeated many times.

The variability discussed above refers only to the intra-laboratory repeatability of measurement, and so relates to the precision of the measurement but not necessarily to its accuracy, and does not deal with inter-laboratory reproducibility. If a variety of laboratories all measure the same sample, the results will scatter and this scatter will likely exceed the scatter of results from a single laboratory. We define the internal and external uncertainty as intra- and inter-laboratory uncertainty. Within the

${ }^{14} \mathrm{C}$ community there has been considerable investment in a series of assessments of laboratory reproducibility (Scott et al., 2003a,b, Scott et al., 2010a,b); at the same time, several studies for ${ }^{10} \mathrm{Be}$ and ${ }^{36} \mathrm{Cl}$ have been performed (Merchel et al., 2011, 2012, Vermeesch et al, 2012).

Proficiency trials are a widely used, international procedure common within the analytical chemistry community that provide a measure of inter-laboratory reproducibility as well as a metric for individual laboratories to assess their performance. From a proficiency test (sometimes called a laboratory intercomparison or a round-robin exercise), an assessment of accuracy, individual laboratory precision (intra-lab, from duplicate samples), and generally, an overall measure of performance (inter-lab, using z-scores) can be achieved. Additionally, material used in the test can form an archive of reference materials, which is valuable for future quality assurance (Thompson et al., 2006, CITAC, 2002). There is a standard literature on this topic but little specifically directed to in-situ cosmogenic isotopes.

The CRONUS-Earth Project had as one of its objectives to rigorously and systematically identify and investigate sources of uncertainty (or variation) in cosmogenic-nuclide production and analysis so as to improve the accuracy and reliability of geochronology techniques and other scientific applications that rely on analyses of cosmogenic nuclides. As part of the CRONUS-Earth project, an analytical inter-comparison was undertaken. Participating laboratories included those funded by CRONUS-Earth and a companion European program, CRONUS-EU. This paper describes the inter-comparison and discusses the results.

Preliminary isotopic data from production rate calibration sites had shown significant variability in results, which may be attributable to different laboratory procedures, variable approaches to data reduction (both half life and assumed standard value), and measurement uncertainty. This laboratory inter-comparison provides an empirical estimate of measurement uncertainty (inter and intra- laboratory) and this is crucial to the overall CRONUS-Earth objectives.

\section{Accuracy and precision}

Measurements are routinely described in terms of their accuracy and/or precision. Measurement accuracy refers to the deviation (difference) of the measured value from the true value (or sometimes the expected or consensus value) while precision 
refers to the variation (expected or observed) in a series of replicate measurements (RSC, 2003). Quality assurance and experimental assessment of these properties occupy much laboratory time through measurement of standards (primary and secondary), reference materials and participation in proficiency trials (RSC, 2005).

Participation in a proficiency trial provides a means of assessing whether the laboratory uncertainty estimate is realistic and whether an accurate measurement can be obtained (through the z-score) in a situation (known as reproducibility conditions) where independent measurements are obtained by the same method on identical samples in different laboratories.

\subsection{Proficiency trials}

Participation in a proficiency trial is often used as a method for assessing the accuracy of laboratories in conducting particular measurements (Thompson et al. 2006). It involves distributing portions of the test material to each laboratory and then analysis of the reported results; this helps each laboratory assess the accuracy and precision of their measurement. The concentrations of the element or isotope in the test materials ideally should be typical of samples routinely measured (Scott et al, $2003 a, b)$. The results from the trial also form the basis of assessment of whether the laboratory uncertainty estimate is realistic (under reproducible conditions), where independent measurements are obtained by the same method on identical samples in different laboratories.

Repeatability refers to measurements made under identical conditions in one laboratory, while reproducibility refers to measurements made in different laboratories, under different conditions. Both repeatability and reproducibility describe the closeness of agreement between the isotope analyses under these two different scenarios and are commonly captured in the standard deviation of the results, which can also be expressed in terms of the coefficient of variation (\% standard deviation) within and between laboratories. The reproducibility standard deviation is the larger of the two terms because it includes both variation within and between the laboratories.

In the CRONUS study, some laboratories provided replicate analyses thus allowing intra-laboratory variability to be assessed, but the focus in this paper is on the interlaboratory variability, which is most pertinent to the isotope user community and on characterising the proposed reference materials. Results have also been expressed in terms of coefficient of variation, allowing comparison across different isotopic activities, and further analysed using z-scores.

\section{Methods}

\subsection{Sample preparation}

We prepared two large $(>20 \mathrm{~kg})$ samples of purified quartz in the University of Vermont cosmogenic isotope laboratories. Sample A was collected by Greg Balco from exposed sandstone outcrops in Antarctica at $77.8830^{\circ} \mathrm{S}, 160.9431^{\circ} \mathrm{E}$, and elevation $1612 \mathrm{~m}$ (Figure 1). The rock pieces were jaw crushed, plate ground, and sieved retaining the $250-800$ micron fraction. Sample A has higher concentrations of cosmogenic nuclides than most samples prepared in laboratories and measured by accelerator mass spectrometry (AMS). Sample N was collected from the beach at sea level from Noosa in Queensland, Australia at $26.4038^{\circ} \mathrm{S}$, $153.1143^{\circ} \mathrm{E}$ (Figure 2). It was sieved to isolate the $250-800$ micron fraction. The nuclide activity in sample $\mathrm{N}$ is typical for samples prepared in many laboratories and routinely measured by AMS. 
We purified quartz using a modified version of the method developed by Kohl and Nishiizumi (1992). The sand-size fractions of both samples were etched twice, overnight in 1-liter teflon bottles using $6 \mathrm{~N} \mathrm{HCl}$ in heated sonicators to remove grain coatings. The etched samples were fed through a roll-type magnetic separator to remove magnetically susceptible minerals. They were then triple etched in 4-liter HDPE bottles under sonication in a heated $\left(70^{\circ} \mathrm{C}\right) 1 \%$ mixture of $\mathrm{HNO}_{3}$ and $\mathrm{HF}$. Every 24 hours, the sample was drained, washed and new acid was added. To further purify the quartz, we re-etched all material in 1-liter HDPE bottles using a $0.5 \%$ mixture of $\mathrm{HNO}_{3}$ and $\mathrm{HF}$ for 72 hours before rinsing the samples repeatedly in deionized water. The etching both purified the samples, removing most minerals other than quartz, and removed meteoric ${ }^{10} \mathrm{Be}$ adhered to the grain surfaces (Figure $3)$.

For sample A, approximately $37 \mathrm{~g}$ of each standard was provided to each participating laboratory, with a recommendation that $5 \mathrm{~g}$ be used per analysis. For sample $\mathrm{N}$, approximately $75 \mathrm{~g}$ was provided to each laboratory, with a recommendation that $20 \mathrm{~g}$ be used per analysis.

Samples were initially shipped in 2008. We have followed up with sample requests since that time, and there is material available to other laboratories on request. Twenty four laboratories in the United States, United Kingdom, France, Switzerland, Germany, the Netherlands, Sweden, Australia and Canada received samples A and $\mathrm{N}$. Each chemical laboratory undertook its own protocols to process the quartz powder to the AMS targets of $\mathrm{BeO}$ and $\mathrm{Al}_{2} \mathrm{O}_{3}$.

\subsection{Statistical analysis}

Summary statistics ( $N$ the number of results, typically greater than the number of laboratories, $\mathrm{n}$ ), the mean, standard deviation (stdev), minimum, maximum and $\mathrm{Q}_{1}$ and $\mathrm{Q}_{3}$ representing the lower $(25 \%)$ and upper $(75 \%)$ quartiles of the distribution are presented. The final summary statistic is the coefficient of variation (CoV) (defined as stdev/mean *100). For simplicity and because of the relatively small number of laboratories, we summarise the variability in the results simply in terms of the overall standard deviation and the CoV.

Formal analysis of the results of the proficiency trial follow an international protocol, involving the use of z-scores (RSC, 2005) based on 2 quantities which need to be calculated or specified. Each laboratory result is converted to a z-score using the following equation

$$
\text { z-score }=\left(\mathrm{X}_{\mathrm{e}-\mathrm{X}} \mathrm{X}\right) / \sigma_{\mathrm{p}}
$$

where $X_{e}$ is the laboratory result, $X_{a}$ is the assessed or known concentration (sometimes called the consensus value). If $X_{a}$ is to be assessed this typically requires about 20 or so laboratories to participate for a robust estimate of the $X_{a}$ value, so that for CRONUS, for some sample/nuclide combination $X_{a}$ has not been formally estimated. Preliminary estimates for $X_{a}$ use the median, since it is a robust measure of the centre of the distribution and is unaffected by extreme values but makes no use of the laboratory quoted error. It has been used in subsequent calculations for the z-score plots. Finally a consensus value calculation based on a weighted average has also been reported (Scott et al, 2003). In these preliminary consensus value calculations, multiple results from a single laboratory are treated as independent. The final component of the z-score calculation is $\sigma_{p}$, which is a standard deviation (sometimes described as being a value 'fit for purpose'). The 
value for $\sigma_{p}(R S C, 2005)$ represents the amount of uncertainty in the results that is tolerable in relation to the purpose of the analysis. In this case, the standard deviation of individual results has been used to estimate $\sigma_{\mathrm{p}}$.

Commonly, the z-scores are interpreted as follows: if the z-scores have values lying between -2 and +2 , the results are considered as acceptable, and values beyond -3 or +3 as representing results that should be further investigated. This is due to a large difference between the measured or consensus value, relative to the $\sigma_{p}$ value. There is no associated uncertainty with a z-score.

\section{Results}

Results were received from varying numbers of laboratories (Table 1) depending on the nuclide. Here, we discuss the set of results (including all replicate sets from the same laboratory), and the number of distinct laboratories that chemically prepared the samples, to distinguish these from the AMS facilities where the samples were measured.

Twenty nine results were obtained for sample $A$, for ${ }^{10} \mathrm{Be}$, which represented 13 different preparation laboratories, using 8 different AMS laboratories and 13 results were obtained for ${ }^{26} \mathrm{Al}$. One set of data that was discrepant was not included in the analysis. Some laboratories reported multiple assays from which we are able to ascertain intra-laboratory variability. The number of results therefore commonly exceeds the number of laboratories since laboratories had been asked to make at minimum duplicate sample preparations (not simply at point of measurement in the AMS) wherever possible. For sample N, 23 results from 11 laboratories were received for ${ }^{10} \mathrm{Be}$, but only 10 sets of results for ${ }^{26} \mathrm{Al}$. The results are shown in Table 2. Samples were measured at AMS facilities of the Lawrence Livermore National Laboratory, Purdue University, SUERC, Uppsala, Arizona, ANSTO, DREAMS and ASTER (Aix-en-Provence).

\subsection{Preliminary and exploratory analysis}

Laboratories provided estimates of isotope concentrations in atoms/g. Additional information routinely provided included the standard to which the analysis was referenced, the value of which depends on the assumed half-life of the nuclide. There is general consensus on the half-life used for ${ }^{26} \mathrm{Al}$, but some variability for ${ }^{10} \mathrm{Be}$. This became an issue because some laboratories had recalculated the NIST standard based on different values for the assumed half life, assuming that the stated ${ }^{10} \mathrm{Be} /{ }^{9} \mathrm{Be}$ ratio of this standard was not correct. However, the stated ratio is the correct value for the NIST standard (Reed, 1990). These differences highlight one of the problems in comparing cosmogenic nuclide data in the past. Tables $2 a$ and $2 b$ shows the complete set of results for each sample; laboratories are identified only by code. As is shown in Table 2, it was necessary to recalculate the results for ${ }^{10} \mathrm{Be}$ to a common basis using the standardization of Nishiizumi et al. (2007). Tables (Tables 3 and 4) for each sample/nuclide, report the summary statistics for the uncorrected and corrected results.

For sample $\mathrm{A},{ }^{10} \mathrm{Be}$, based on the replicate results reported by individual laboratories, we can determine that the intra-laboratory CoV ranged from 0.3 to $2 \%$; for sample $\mathrm{N}$, $\mathrm{Be}-10$, the intra-laboratory CoV ranged from 0.3 to $4.3 \%$. 
As part of the study of the inter-laboratory variability, we asked laboratories to identify the basis of their standardization and were able to correct discrepancies by ensuring that all data was normalized to the value of the NIST standard given by Nishiizumi et al. (2007) of $2.79 \times 10^{-11}{ }^{10} \mathrm{Be} /{ }^{9} \mathrm{Be}$ (07KNSTD). The reader should note that this differs slightly from the original normalization of the NIST ${ }^{10} \mathrm{Be}$ standard (Reed 1990), which stated a ratio of $2.68 \times 10^{-11}{ }^{10} \mathrm{Be} /{ }^{9} \mathrm{Be}$. The values of the NIST and KNST standards are taken as their reported atomic ratios. Both the unadjusted and adjusted summary statistics are shown in Tables $3 a$ and $4 a$. Correction to the common standard reduced the coefficient of variation for sample $A$ for the ${ }^{10} \mathrm{Be}$ analyses to $2.9 \%$. For sample $\mathrm{N}$, which has two orders of magnitude lower concentration (see table 3 ), the coefficient of variation is approx $4.1 \%$. Tables $3 \mathrm{~b}$ and $4 \mathrm{~b}$ show the same summary statistics for ${ }^{26} \mathrm{Al}$, but with higher $\mathrm{CoV}$ values than for the corrected ${ }^{10} \mathrm{Be}$ results.

The more formal exploratory analysis proceeded by calculating and summarising the z-scores for each laboratory on each sample. These are shown graphically in Figures 4 and 5 . For the z-score analysis we have used the median as the assessed value. From Figures 4 and 5 , the majority of $z$-scores lie in the range of -2 to +2 , with some exceptions, predominantly in ${ }^{10} \mathrm{Be}$ measurements and for sample $\mathrm{A}$. Considering all the $\mathrm{Z}$-values for both samples $A$ and $N$ and both isotopes, the vast majority of values lie in the range -3 to +3 , and would not pose a "cause for concernresults which are anomalous" under standard international proficiency trial guidelines (RSC 2005). The small number (7 out of 67 or 10\%) of results outside of this range might be considered as anomalous (or outliers). This is very similar to the percentage anomalous values identified in the ${ }^{14} \mathrm{C}$ inter-comparison studies (Scott et al, 2010a,b) that are based on organic materials.

To provide a preliminary consensus value for ${ }^{10} \mathrm{Be}$ for samples $\mathrm{A}, \mathrm{N}$ a weighted average (and its uncertainty) has been calculated (Scott et al, 2003). This has not been done for ${ }^{26} \mathrm{Al}$ because of the small number of results. This is summarised Table 5 .

\section{In-situ ${ }^{14} \mathrm{C}$ measurement on sample $\mathrm{A}$}

Twenty three measurements of cosmogenic ${ }^{14} \mathrm{C}$ in sample $\mathrm{A}$ were made by a total of 4 laboratories: the University of Arizona, Purdue University, ETH Zurich, and LamontDoherty Earth Observatory. Results are shown in Table 6.

The ratio of ${ }^{14} \mathrm{C} /{ }^{10} \mathrm{Be}$ in sample $\mathrm{A}$ can be estimated to be $0.0203 \pm 0.0016$. Sample $\mathrm{A}$ was collected in Antarctica and can be assumed to be from a saturated surface with negligible erosion. The cosmogenic ${ }^{14} \mathrm{C}$ result for sample $\mathrm{A}$ is reasonable, since the production ratio ${ }^{14} \mathrm{C} /{ }^{10} \mathrm{Be}$ from fast neutrons should be 2.93 (Jull et al. 1989) based on neutron irradiations.

\section{Discussion and further work}

This paper reports on a laboratory inter-comparison for the cosmogenic nuclides ${ }^{26} \mathrm{Al}$ and ${ }^{10} \mathrm{Be}$, using natural samples, with concentrations that differ by two orders of magnitude. Laboratories were not constrained to use common procedures or standards. Employing different standards created a source of variability in the results, however, after normalization, that variability could be reduced. We strongly encourage the use of agreed standards and normalization procedures in cosmogenic nuclide work. In particular, ${ }^{10} \mathrm{Be}$ should be normalized using the values given by Nishiizumi et al. (2007). 
Inter-comparisons allow an empirical estimate of measurement uncertainty (both inter- and intra-laboratory). The variability amongst replicates is smaller in the vast majority of cases than variability between laboratories. With regard to the desired precision of less than $5 \%$, our results to date indicate that we can constrain the interlaboratory ${ }^{10} \mathrm{Be}$ errors below $5 \%$ for materials with high ${ }^{10} \mathrm{Be}$ concentrations but that the scatter in ${ }^{26} \mathrm{Al}$ measurements is larger. Variability of in-situ ${ }^{14} \mathrm{C}$ analyses is similar to the inter lab variability as found for ${ }^{26} \mathrm{Al}$.

It is unlikely that this variability is related to sample inhomogeneity because each sample contained thousands to tens of thousands of individual quartz grains that were well and repeatedly mixed during quartz purification. Extraction procedures differ between laboratories and most importantly, each laboratory uses different carriers to spike each sample of quartz in what is an isotope dilution experiment. Determining the ${ }^{9} \mathrm{Be}$ concentration in beryllium carriers used by each lab is critical to the final measured value of ${ }^{10} \mathrm{Be}$ since it is the ratio ${ }^{10} \mathrm{Be} /{ }^{9} \mathrm{Be}$ is measured by AMS. Similarly, the accuracy and precision by which ${ }^{27} \mathrm{Al}$ is measured $\left({ }^{27} \mathrm{Al}\right.$ is native to the quartz) directly affects the reported concentration of ${ }^{26} \mathrm{Al}$.

Good practical QA procedures are routine in many laboratories including measurement of replicates and use of standards. The cosmogenic measurement community is still rather small, so that a relatively small number of laboratories participated, although this does represent a large proportion of those laboratories focusing on in situ cosmogenic isotope work. Results have shown the existence of significant inter-laboratory variability, and the role the inter-comparisons to provide an empirical estimate of measurement uncertainty (inter and intra- laboratory) is crucial for the future of CRONUS-related work.

For the two samples and for ${ }^{10} \mathrm{Be}$, we have provided consensus values which can then be used as reference values for these two materials. We have not calculated consensus values for ${ }^{26} \mathrm{Al}$ or ${ }^{14} \mathrm{C}$ due to the small number of results (although the median will provide a working value). Further work to characterise the materials by defining the consensus values would be required.

A substantial archive of material has also been created at the University of Arizona, which is an important outcome of the project, since this will be invaluable in further work. The value of the archive depends on the quality of the test materials and on the willingness of labs to participate. For CRONUS, the number of laboratories participating was limited but the value of these reference materials will increase as the materials are routinely measured. Further samples will be added to the archive in future for distribution on request to laboratories. We have already distributed additional intercomparison materials for these and will expand the study to other nuclides.

Like other isotope measurement communities (such as ${ }^{14} \mathrm{C}$ ), there is very clearly a need for a longer-term inter-comparison program such as those in other communities, and which will grow the number of reference materials as well as informing the user communities of realistic uncertainties.

\section{Acknowledgements}

This work was funded as part of the CRONUS_Earth program by the US National Science Foundation, grant EAR0345150. 


\section{References}

Jull, A. J. T., Englert, P. A. J., Donahue, D. J., Reedy, R. C. and Lal, D. 1989. Cosmogenic nuclide production rates: C14 from neutron spallation. Lunar and Planetary Science 20, 490-491.

Kohl, C. P. and Nishiizumi, K., 1992, Chemical isolation of quartz for measurement of in-situ -produced cosmogenic nuclides: Geochim.

Cosmochim. Acta, 56, 3583-3587.

Merchel S., Bremser W., Alkhmadaliev S., Arnold M., Aumaitre G., Bourles, D. L., Braucher, R., Caffee, M., Christl, M., Fifield, L. K., Finkel, R. C., Freeman, S. P. H. T., Kubik, P., Martschini, M., Rood, D. H., Ruiz-Gomez, A., Tims, S. G., Wallner, A., Wilcken, K. M. and Xu, S. (2012) Quality assurance in accelerator mass spectrometry: results from an international round-robin exercise for Be-10. Nucl. Instrum. Meth. Phys. Res. B 289, 68-73.

Merchel S, Bremser W, Alfimov M, Aumaitre G, Benedetti L, Bourles D L, Caffee M, Fifiled L K, Finkel T C, Freeman S P H T, Martschini M, Matsushi Y, Rood D H, Sasa K, Steier P, Takahashi T, Tamari M, Tims S G, Tosaki Y, Wilcken K M, Xu S. (2011) Ultra trace analysis of $\mathrm{Cl}-36$ by accelerator mass spectrometry: an interlaboratory study. Anal. Bioanal. Chem. 400, 3125-3132.

Nishiizumi, K., Imamura, M., Caffee, M. W., Southon, J. S., Finkel, R. C. and McAninch, J. 2007. Absolute calibration of ${ }^{10} \mathrm{Be}$ AMS standards. Nucl. Instrum. Meth. Phys. Res. B, 258, 403-413.

Reed, W. P. 1990. Standard Reference Material 4325 Certificate, National Institute of Standards and Technology. https://www.nist.gov/srmors/certificates/4325.pdf Retrieved 23 October 2012.

Royal Society of Chemistry 2003. Technical brief 13. Part 1: Accuracy, precision and uncertainty

Royal Society of Chemistry 2005. Technical brief $18 a$, What is proficiency testing? Guide for end-users of chemical data. http://www.rsc.org/images/proficiency-testingtechnical-brief-18A tcm18-214885.pdf.

Scott, E. M. 2003a. The Third International Radiocarbon Intercomparison.

Radiocarbon 45(2), 253-328.

Scott E M. 2003b. The Fourth International Radiocarbon Intercomparison.

Radiocarbon 45(2), 135-252.

Scott, E.M., Cook, G., and Naysmith, P. 2010a. The fifth international radiocarbon intercomparison (VIRI): An assessment of laboratory performance in stage 3. Radiocarbon, 53 (2-3). pp. 859-865.

Scott, E.M., Cook, G.T., and Naysmith, P. 2010b. A report on phase 2 of the Fifth International Radiocarbon Intercomparison (VIRI). Radiocarbon, 52 (3). 846-858.

Thompson M, Ellison S, Wood R 2006. The international harmonized protocol for the proficiency testing of analytical chemistry laboratories. Pure Appl. Chem., Vol. 78, No. 1, 145-196.

CITAC/Eurochem 2002. Guide to Quality in Analytical Chemistry

Taverniers I, de Loos M, van Bockstaele E 2004. Trends in quality in the analytical laboratory. II. Analytical method validation and quality assurance Trends in Anal. Chem. 23, No. 8, 535-552.

Vermeesch P, Balco G, Blard P-H, Dunai T, Kober F, Niedermann S, Shuster D L, Strasky S, Stuart F M, Wieler R, Zimmerman L (2012) Interlaboratory comparison of cosmogenic ${ }^{21} \mathrm{Ne}$ in quartz. Quaternary Geochronology, 1-9. 
Table 1: Participating Laboratories

Scottish University Environmental Research Centre (SUERC), Glasgow, Scotland CEREGE, Collège de France, Aix-en Provence, France.

PRIME Lab, Purdue University, West Lafayette, Indiana USA

Department of Geography, University of Edinburgh, Scotland

School of Geographical and Earth Sciences, University of Glasgow, Glasgow

Uppsala University, Sweden.

University of Washington, Seattle, Washington USA

ASTER Laboratory, Aix-en-Provence, France

Australian National Science and Technology Organisation (ANSTO), Sydney,

Australia.

Lamont-Doherty Earth Observatory, Palisades, NY USA

Lawrence Livermore National Laboratory, Livermore, California, USA

University of Arizona, Tucson, Arizona USA

University of California-Berkeley, California, USA

DREAMS Laboratory, Dresden, Germany

Table 3a. Summary results for sample A, ${ }^{10} \mathrm{Be}$ uncorrected (1) and corrected (2)

\begin{tabular}{|l|l|l|l|l|l|l|l|l|l|}
\hline $\mathrm{N}$ & $\mathrm{n}$ & mean & stdev & $\min$ & $\mathrm{Q} 1$ & Median & $\mathrm{Q} 3$ & $\max$ & $\mathrm{CoV}$ \\
\hline 29 & 13 & $3.52 \mathrm{E}+07$ & $0.17 \mathrm{E}+07$ & $3.26 \mathrm{E}+07$ & $3.43 \mathrm{E}+07$ & $3.49 \mathrm{E}+07$ & $3.6 \mathrm{E}+07$ & $3.86 \mathrm{E}+07$ & $4.8 \%{ }^{(1)}$ \\
\hline 29 & 13 & $3.42 \mathrm{E}+07$ & $0.1 \mathrm{E}+07$ & $3.16 \mathrm{E}+07$ & $3.33 \mathrm{E}+07$ & $3.45 \mathrm{E}+07$ & $3.48 \mathrm{E}+07$ & $3.86 \mathrm{E}+07$ & $2.9 \%^{(2)}$ \\
\hline
\end{tabular}

Table 3b. Summary results for sample $A,{ }^{26} \mathrm{Al}$

\begin{tabular}{|l|l|l|l|l|l|l|l|l|l|}
\hline $\mathrm{N}$ & $\mathrm{n}$ & mean & stdev & $\min$ & $\mathrm{Q} 1$ & Median & Q3 & $\max$ & CoV \\
\hline 13 & 7 & $1.43 \mathrm{E}+08$ & $7.0 \mathrm{E}+06$ & $1.24 \mathrm{E}+08$ & $1.4 \mathrm{E}+08$ & $1.44 \mathrm{E}+08$ & $1.46 \mathrm{E}+08$ & $1.54 \mathrm{E}+08$ & $4.9 \%$ \\
\hline
\end{tabular}

Table 4a. Summary results for sample $\mathrm{N},{ }^{10} \mathrm{Be}$

\begin{tabular}{|l|l|l|l|l|l|l|l|l|l|}
\hline $\mathrm{N}$ & $\mathrm{n}$ & mean & stdev & $\min$ & $\mathrm{Q} 1$ & Median & $\mathrm{Q} 3$ & $\max$ & $\mathrm{CoV}$ \\
\hline 23 & 12 & $2.26 \mathrm{E}+05$ & $1.6 \mathrm{E}+04$ & $2 \mathrm{E}+05$ & $2.16 \mathrm{E}+05$ & $2.22 \mathrm{E}+05$ & $2.42 \mathrm{E}+05$ & $2.58 \mathrm{E}+05$ & $7.1 \%{ }^{(1)}$ \\
\hline 23 & 12 & $2.17 \mathrm{E}+05$ & $0.88 \mathrm{E}+04$ & $2 \mathrm{E}+05$ & $2.1 \mathrm{E}+05$ & $2.17 \mathrm{E}+05$ & $2.22 \mathrm{E}+05$ & $2.35 \mathrm{E}+05$ & $4.1 \%^{(2)}$ \\
\hline
\end{tabular}

Table 4b. Summary results for sample $\mathrm{N},{ }^{26} \mathrm{Al}$

\begin{tabular}{|l|l|l|l|l|l|l|l|l|l|}
\hline $\mathrm{N}$ & $\mathrm{n}$ & mean & stdev & min & $\mathrm{Q} 1$ & Median & Q3 & max & CoV \\
\hline 10 & 5 & $1.05 \mathrm{E}+06$ & $1.06 \mathrm{E}+05$ & $8.6 \mathrm{E}+05$ & $9.9 \mathrm{E}+05$ & $1.06 \mathrm{E}+06$ & $1.10 \mathrm{E}+06$ & $1.24 \mathrm{E}+05$ & $10.1 \%$ \\
\hline
\end{tabular}

Table 5: Preliminary consensus values for ${ }^{10} \mathrm{Be}$ for samples $\mathrm{A}$ and $\mathrm{N}$ and 1 sigma uncertainty

\begin{tabular}{|l|l|l|}
\hline Sample & Consensus value & $1 \sigma$ \\
\hline A & $3.303 \mathrm{E}+07$ & $7.446 \mathrm{E}+04$ \\
\hline N & $2.075 \mathrm{E}+05$ & $1.194 \mathrm{E}+03$ \\
\hline
\end{tabular}

Table 6. Summary results for sample $\mathrm{A},{ }^{14} \mathrm{C}$

\begin{tabular}{|l|l|l|l|l|l|l|l|l|l|}
\hline $\mathrm{N}$ & $\mathrm{n}$ & mean & stdev & $\min$ & $\mathrm{Q} 1$ & median & $\mathrm{Q} 3$ & $\max$ & $\mathrm{CoV}$ \\
\hline 23 & 4 & $6.93 \mathrm{E}+05$ & $4.4 \mathrm{E}+04$ & $5.9 \mathrm{E}+05$ & $6.63 \mathrm{E}+05$ & $6.97 \mathrm{E}+05$ & $7.23 \mathrm{E}+05$ & $7.74 \mathrm{E}+05$ & $6.34 \%$ \\
\hline
\end{tabular}


Table $6 \mathrm{~b}$ Summary results for sample A, number of ${ }^{14} \mathrm{C}$ atoms by laboratory

\begin{tabular}{|l|l|l|l|l|}
\hline & A & B & C & $D$ \\
\hline$n$ & 7 & 6 & 8 & 2 \\
\hline mean & $7.25 \mathrm{E}+05$ & $6.51 \mathrm{E}+05$ & $6.99 \mathrm{E}+05$ & $6.80 \mathrm{E}+05$ \\
\hline stdev & $3.594 \mathrm{E}+04$ & $3.269 \mathrm{E}+04$ & $4.007 \mathrm{E}+04$ & $*$ \\
\hline median & $7.33 \mathrm{E}+05$ & $6.60 \mathrm{E}+05$ & $7.00 \mathrm{E}+05$ & $6.80 \mathrm{E}+05$ \\
\hline Cvar & 4.96 & 5.02 & 5.73 & $*$ \\
\hline
\end{tabular}


Figure legends.

Figure 1. Collection of sample A from Antarctic sandstone outcrop by G. Balco.

Figure 2. Beach at Noosa, Queensland, Australia from which sample N was collected.

Figure 3. The cation content of quartz prepared as standards, which reflects the presence of non-quartz impurities, decreased with repeated etching. A. N standard. B. A Standard. Data from HF dissolution of aliquots removed during different processing steps. Chemistry determined by ICP-OES analyses.

Figure 4: $\mathrm{z}$-scores for ${ }^{10} \mathrm{Be}$ results for (a) samples $\mathrm{A}$ and (b) $\mathrm{N}$ for individual laboratories

Figure 5. z-scores for ${ }^{26} \mathrm{Al}$ results for (a) samples $\mathrm{A}$ and (b) $\mathrm{N}$ for individual laboratories 